R. Schön · R. Gutwald · A.Schramm · G. Nils-Claudius · R. Schmelzeisen

Klinik und Poliklinik für Mund-, Kiefer- und Gesichtschirurgie, Universitätsklinik, Albert-Ludwigs-Universität Freiburg

\title{
Extraorale und intraorale endoskopisch assistierte Versorgung von Kollumfrakturen
}

tion nach lateral. Der extraorale Zugang war bei stark dislozierten Kollumfrakturen mit Dislokation nach medial oder bei Trümmerfrakturen indiziert.

\section{Schlüsselwörter}

Kollumfraktur · Endoskopisch assistierte Osteosynthese · Extraoral · Transoral

Patienten und Methoden: Die endoskopisch assistierte operative Versorgung von Kollumfrakturen wurde bei 17 unselektierten Patienten, bei denen in dem Zeitraum von April 1998-Dezember 1999 die Indikation zur operativen Versorgung von Kollumfrakturen gestellt wurde, durchgeführt. 14 der $17 \mathrm{~Pa}$ tienten zeigten weitere Unterkieferfrakturen, 11 der operativ versorgten Kollumfrakturen waren disloziert, 7 nichtdisloziert. Bei 1 Patientin wurde eine bilaterale dislozierte Kollumfraktur operativ behandelt. Eine nichtdislozierte Kollumfraktur war nach offener Reposition und Osteosynthese einer Trümmerfraktur des aufsteigenden Unterkieferasts der Gegenseite auffällig und wurde konservativ behandelt.

Zugänge: 9 der 17 Patienten wurden über einen submandibularen, 8 über einen transoralen Zugang operativ versorgt. Eine regelrechte Frakturreposition und Osteosynthese wurden über den submandibularen und den transoralen Zugang mit endoskopisch assistierter Technik erreicht. Bei 4 von den $8 \mathrm{~Pa}$ tienten, die über einen transoralen Zugang versorgt wurden, wurde unter Verwendung von gewinkelten Bohrern und Schraubendrehern die Osteosynthese transoral ohne transbukkale Stichinzision durchgeführt. Der transorale Zugang bewährte sich auch als zuverlässiger Zugang bei der operativen Versorgung von Kollumfrakturen mit Disloka-

\section{Collumfrakturen treten mit einer Häu-} figkeit von 9-45\% aller Unterkieferfrakturen auf $[16,17,18]$. Die konservative Behandlung ist auch bei dislozierten Kollumfrakturen weit verbreitet [20]. Die anatomische Reposition insbesondere bei dislozierten Frakturen wird selten erreicht. Eine adäquate Rehabilitation und Kiefergelenkfunktion wird durch eine forcierte Adaption bei einer veränderten Kollummorphologie nicht immer erreicht.

Für die operative Behandlung von Kollumfrakturen werden unterschiedliche Techniken beschrieben. Die Miniplattenfixation hat sich als eine Technik zur Stabilisierung des Repositionsergebnisses und zur Vermeidung einer intermaxillaren Fixation bewährt $[4,9]$ (Abb. 1, 2). Für die offene Frakturreposition über einen präaurikulären, retromandibularen oder submandibularen Zugang wird das Risiko der N.-facialisSchädigung und von Narben im sichtbaren Bereich beschrieben [21, 23]. Die Indikationen für eine offene Reposition oder die konservative Frakturbehand- lung werden aufgrund dieser möglichen Komplikationen kontrovers diskutiert $[4,7,20,23]$. Unter Verwendung eines Endoskops wird nach begrenzten Inzisionen in Bereichen limitierter Exposition eine verbesserte Sicht erreicht. Das Risiko einer Schädigung des N. facialis und das von Narben im sichtbaren Bereich können über diese minimalinvasive Technik reduziert werden $[11,12,15]$. Bei einem rein transoralen Zugang besteht bei subperiostaler Präparation kein Risiko für eine Schädigung des N. facialis. Narben im sichtbaren Bereich werden bei diesem operativen Zugang vermieden $[1,8,15]$.

Die Indikation für die operative extraorale und transorale endoskopisch assistierte Versorgung von Kollumfrakturen wird demonstriert.

\section{Material und Methode}

Bei 17 Patienten wurde die endoskopisch assistierte, operative Versorgung von Kollumfrakturen durchgeführt. 14 der 17 Patienten zeigten weitere Unterkieferfrakturen. 12 Patienten waren männlich, 5 weiblich mit einem mittleren

Online publiziert: 7 Februar 2002

(c) Springer-Verlag 2002

Dr.Dr. Ralf Schön

Klinik und Poliklinik

für Mund-, Kiefer- und Gesichtschirurgie,

Universitätsklinik,

Albert-Ludwigs-Universität Freiburg, Hugstetter Straße 55, 79106 Freiburg, E-mail:schoen@zmk2.ukl.uni-freiburg.de, Phone:0761-2704919, Fax:0761-2704900 
R. Schön · R. Gutwald · A. Schramm • G. Nils-Claudius $\cdot$ R. Schmelzeisen

\section{Extraoral and intraoral endoscopically assisted treatment of condylar mandible fractures}

\section{Abstract}

Aim of the study: Using limited extraoral and transoral incisions for an endoscopically assisted open reduction of condylar mandible fractures, the risk of facial nerve damage and extensive visible scars can be reduced.

Patients and methods: The endoscopically assisted treatment of 17 consecutive patients with fractures of the condyle was performed from April 1998 to December 1999. Of the 17 patients, 14 had additional mandibular fractures, and 11 of the condylar fractures were dislocated. Of the 17 patients, 9 were treated by submandibular and 8 by transoral approach. Adequate anatomic reduction was achieved by the submandibular and transoral approaches using an endoscopically assisted technique.

Approaches: In four patients angulated drills and screwdrivers facilitated the transoral treatment of condylar fractures. Transbuccal stab incisions and the use of trocars were not needed in these four patients. The transoral approach proved to be a reliable surgical approach for fractures of the mandibular condyle even when dislocation with lateral override was present. The extraoral approach was used for severely dislocated fractures such as fractures with medial override or comminution.

\section{Keywords}

Fracture of the collum · Endoscopically assisted osteosynthesis · Extraoral · Transoral
Durchschnittsalter von 32 Jahren. Der Frakturtyp, das Maß der Dislokation und das Ergebnis der operativen Reposition wurden intraoperativ endoskopisch und prä- und postoperativ röntgenologisch mit Panoramaschichtaufnahmen und Unterkiefer-PA-Aufnahmen nach Clementschitsch festgestellt. 6 Monate postoperativ wurden die Unterkiefer- und Kiefergelenkfunktion durch Untersuchung der maximalen Mundöffnung, der Deviation bei der Mundöffnung, der Latero- und Protrusion, Kiefergelenkknacken und Kiefergelenkbeschwerden untersucht. In der extraoralen Gruppe wurden die Länge, das Erscheinungsbild der sichtbaren Narben und die Funktion des N. facialis evaluiert.

\section{Endoskopieequipment}

Endoskope mit einem Durchmesser von $4 \mathrm{~mm}, 30^{\circ}$-Optiken (Karl Storz, Tuttlingen, Deutschland) und eine Xenonlichtquelle wurden verwendet. Mit einer Spül-Saug-Vorrichtung wurden eine gute Übersicht in den limitierten optischen Kavitäten und eine zeitsparende Reinigung der Linse erreicht.

\section{Chirurgische Technik}

Bei dem submandibularen Zugang wurde nach Durchtrennung des Platysmas der aufsteigende Ast des Unterkiefers dargestellt. Streng subperiostal wurde endoskopisch assistiert die Präparation nach kranial fortgeführt, bis die Fraktur im Endoskop zur Darstellung kam.

Bei dem transoralen Zugang wurde im Sinn einer Schnittführung, wie sie für die sagittale Spaltung des Unterkiefers durchgeführt wird, vorgegangen. Der Unterkiefer wurde subperiostal bis zum Kieferwinkel dargestellt. Um eine gute Übersicht zu erreichen, wurden inferiore Anteile des Ansatzes des M. temporalis vom Proc. muscularis abgelöst. Dann wurde subperiostal die optische Kavität nach kranial präpariert, bis die Fraktur im Endoskop sichtbar wurde. Bei der streng subperiostalen Präparation werden eine Verletzung des M. masseter vermieden und eine gute Übersicht ohne Blutung in der optischen Kavität erreicht.

Unter endoskopischer Sicht wurden die Präparation der Weichgewebe durchgeführt und eine 2,0-AO/ASIF-Mini- platte (DCP-Synthes Paoli, PA, USA) mit mindestens 2 Schrauben beidseits der Fraktur fixiert (Abb. 3).

\section{Frakturreposition und Fixation}

Zur Erleichterung der Reposition der Kollumfraktur wurde die Gelenkregion beim submandibularen Zugang mit einer Frakturrepositionszange im Bereich des Kieferwinkels und beim transoralen Zugang durch Druck auf die Molaren im Unterkiefer distrahiert. Mit modifizierten Raspatorien wurden die Frakturreposition durchgeführt und die Schraubeninsertion über die transbukkale Stichinzision erreicht. Nach Reposition wurde das Fragment durch Druck mit einem Raspatorium auf die laterale Oberfläche stabilisiert, und eine Miniplatte wurde mit einer Schraube im proximalen Fragment frakturnah fixiert. Durch Zug an der Miniplatte nach kaudal wurde die Reposition erleichtert.

Nach der Reposition wurde die Miniplatte mit einer 2. Schraube frakturnah im aufsteigenden Ast verankert, bevor die Osteosynthese vervollständigt wurde. Die achsengerechte Reposition wurde endoskopisch insbesondere im Bereich des posterioren Rands des aufsteigenden Asts kontrolliert.

Bei 4 Patienten mit transoralem $\mathrm{Zu}$ gang wurde die Osteosynthese mit abgewinkelten Bohrern und Schraubendrehern rein transoral durchgeführt, um transbukkale Stichinzisionen zu vermeiden (Abb. 4).

\section{Ergebnis}

Bei 17 Patienten wurde die operative Versorgung von Kollumfrakturen endoskopisch assistiert durchgeführt. Der submandibulare Zugang wurde bei $9 \mathrm{~Pa}$ tienten, der transorale Zugang bei $8 \mathrm{~Pa}-$ tienten angewendet. 9 Patienten, die über den submandibularen Zugang operiert wurden, zeigten 7 mittelhohe und 2 tiefe Kollumfrakturen und 1 Trümmerfrakturen der Gelenkfortsatzbasis. Eine Patientin zeigte eine stark dislozierte beidseitige mittelhohe Kollumfraktur (Abb. 2,3).

In der extraoralen Gruppe waren 7 von 11 operativ versorgten Frakturen disloziert, 3 zeigten eine ausgeprägte mediale Dislokation mit Verlagerung des Fragments um etwa $90^{\circ}$, und 2 Frakturen zeigten ein mediales Override. 
Abb. 1a-c $>$ Panoramaschichtaufnahme nach transoraler, endoskopisch assistierter Reposition und Fixation einer dislozierten lamellären Kollumfraktur mit lateraler Dislokation: prä- (a), postoperativ (b) und nach Metallentfernung 9 Monate postoperativ (c)
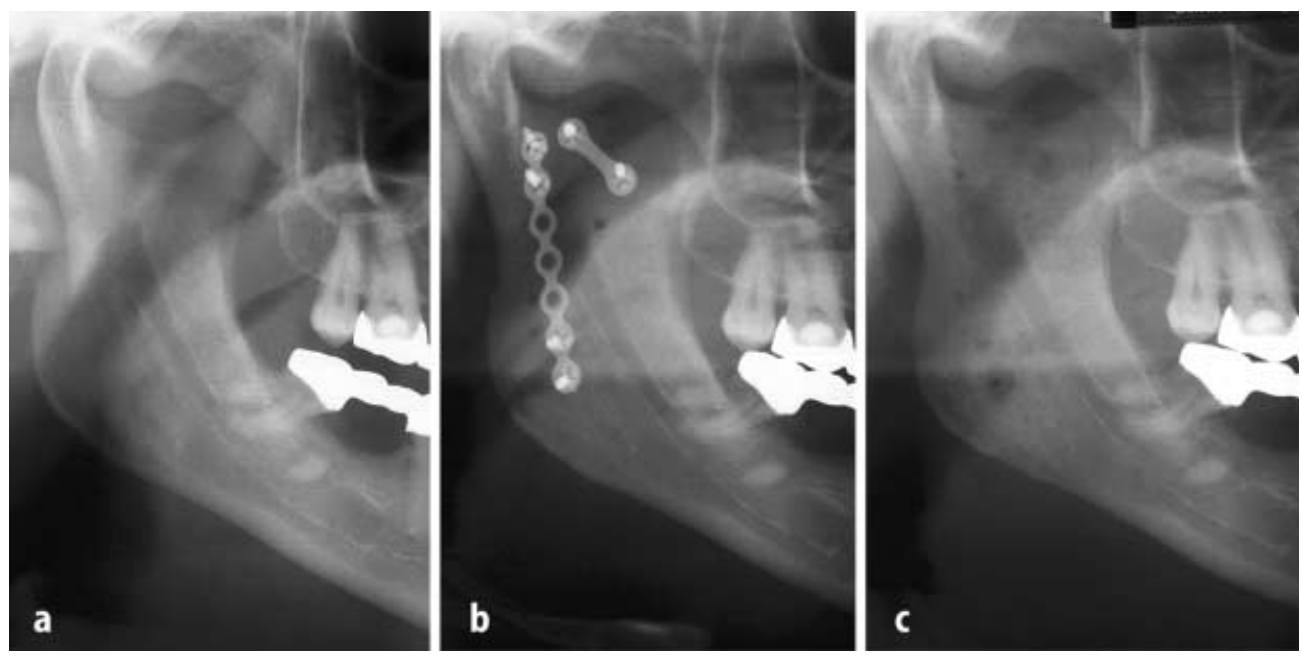

8 Patienten, die über einen transoralen Zugang operiert wurden, wiesen 3 mittelhohe Kollumfrakturen und 5 tiefe Kollumfrakturen auf. In der Gruppe, die von transoral operiert wurde, waren 2 von 8 Frakturen disloziert.

Zur Osteosynthese der Kollumfrakturen wurden 2,0-Zygoma-4- oder 5Loch-AO-ASIF-Miniplatten verwendet (Synthes, Paoli, PA, USA). Bei tiefen Kollumfrakturen wurde, wenn möglich, eine weitere 2,0-Miniplatte verwendet (Abb. 1). Die Reposition und die Fixation wurden durch die endoskopische Visualisierung erleichtert. Transbukka- le Stichinzisionen für die Schraubeninsertion wurden bei allen Patienten mit submandibularem Zugang und bei 4 von 8 Patienten mit einem transoralen Zugang durchgeführt. Bei 4 Patienten wurde unter Verwendung von abgewinkelten Bohrern und Schraubendrehern die Osteosynthese ohne transbukkale Stichinzision durchgeführt (Abb. 4). Eine temporäre Schwäche des R. mandibulae des N. facialis war bei 2 Patienten mit submandibularem Zugang postoperativ auffällig. Nach guten Erfahrungen mit abgewinkelten Bohrern und Schraubendrehern zur transoralen Metallent- fernung bei Kollumfrakturen, die von extraoral versorgt wurden, wurde die Osteosynthese mit abgewinkelten Bohrern und Schraubendrehern bei den letzten 4 Patienten der vorliegenden Studie rein transoral durchgeführt. Bei diesen 4 Patienten mit transoralem Zugang wurden somit transbukkale Stichinzisionen vermieden. Bei 2 Patienten, die von transoral ohne transbukkale Stichinzision versorgt wurden, zeigte sich intraoperativ eine ausgeprägte Frakturdislokation. Bei einer dieser beiden Frakturen war die Reposition durch den lamellären Verlauf der Fraktur und die
Abb. 2a-d Dislozierte beidseitige Kollumfraktur von submandibular. Panoramaschichtaufnahme vor (a) und nach (b) endoskopisch assistierter Reposition und Fixation, $c, d$ in der präoperativen Unterkiefer-

PA-Aufnahme nach Clementschitsch ist eine bilaterale Verkürzung des aufsteigenden Unterkieferasts bei medialer Frakturdislokation auffällig. Nach endoskopisch assistierter Reposition und Fixation zeigt sich eine achsengerechte Stellung der Fragmente
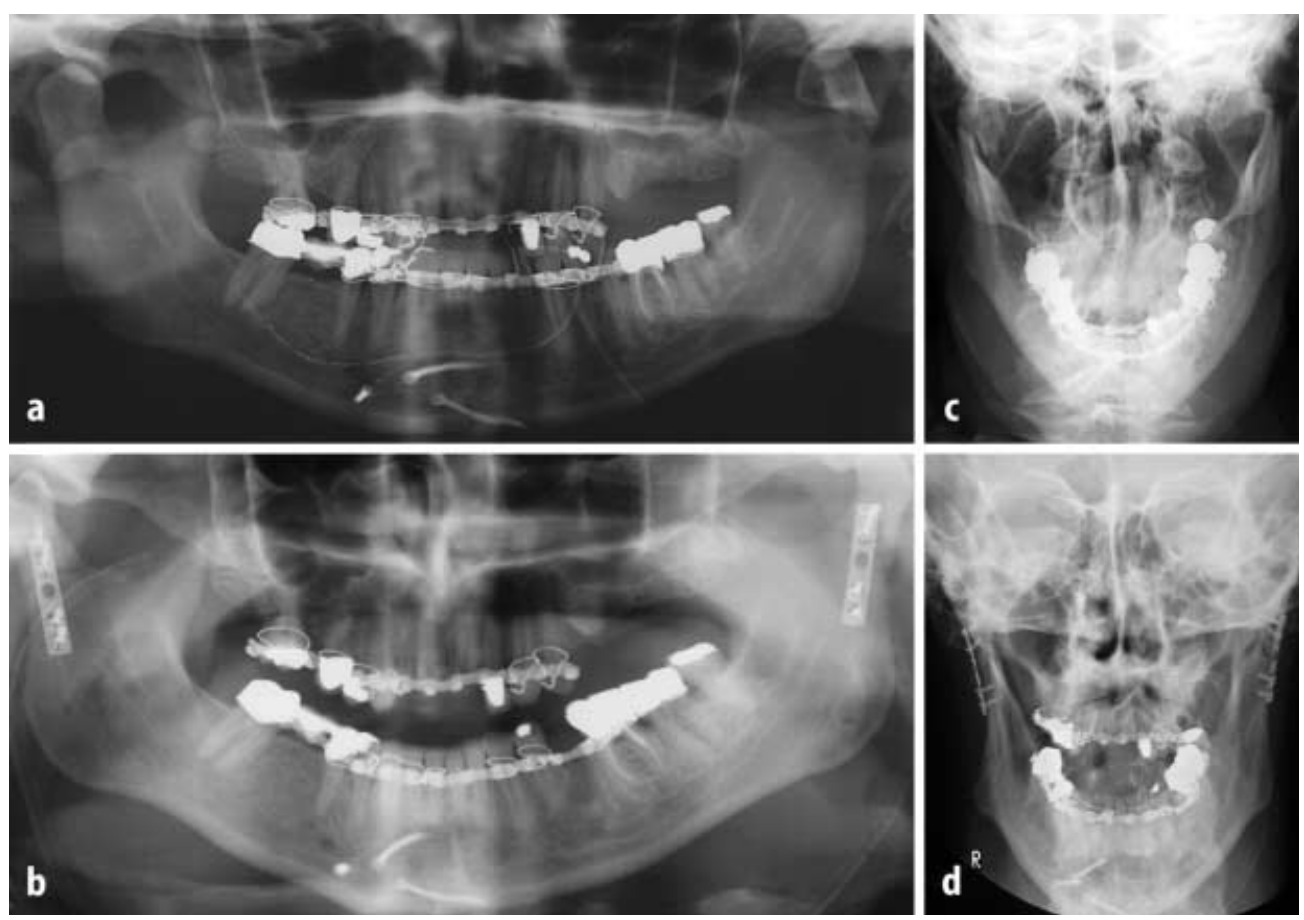


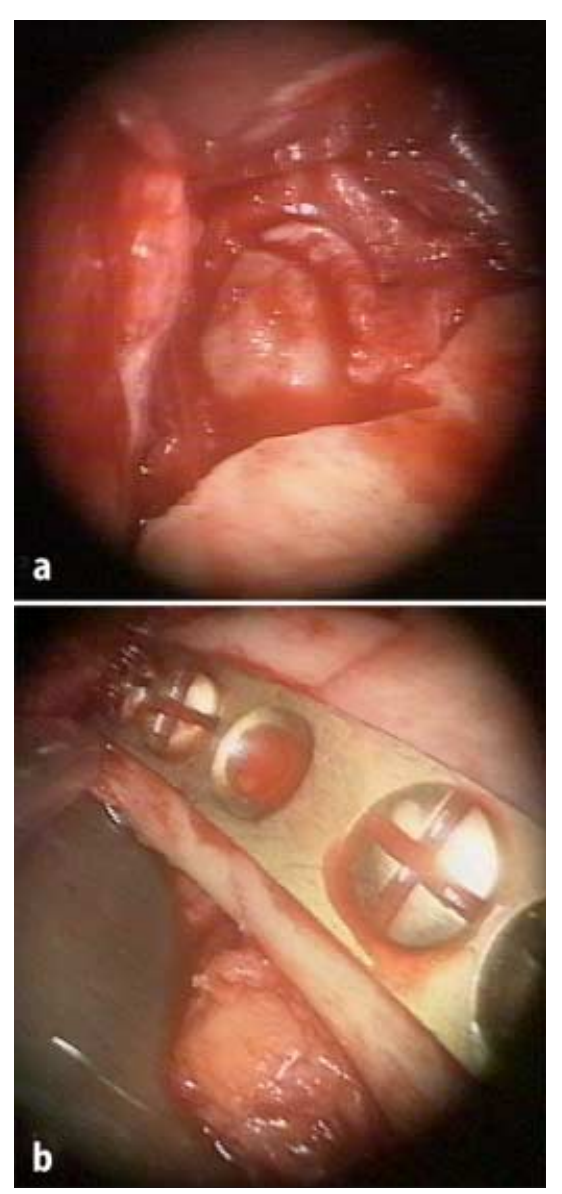

Abb. $3 \Delta$ Intraoperatives Bild der Patientin aus Abb. 2, Frakturdislokation des rechten Fragments mit "medial override" mit einer $30^{\circ}-0 \mathrm{p}$ tik (Karl Storz, Tuttlingen). Das Kiefergelenkköpfchen ist außerhalb der Gelenkpfanne. Nach Reposition und Osteosynthese mit einer 2,0AO/ASIF-Zygoma-Miniplatte aus Titan ist die intraoperative, endoskopische Kontrolle der achsengerechten Reposition am Hinterrand des aufsteigenden Asts dargestellt

Aussprengung eines Fragments am proximalen Frakturrand erschwert (Abb. 1). Das rein transorale Vorgehen unter Verwendung von abgewinkelten Bohrern und Schraubendrehern hat sich in unserer Klinik bewährt und wird seit 1999 routinemäßig eingesetzt.

Die achsengerechte anatomische Frakturreposition wurde auch bei dieser anspruchsvollen dislozierten Frakturen rein transoral durchgeführt (Abb. 1,2,3). Eine adäquate anatomische Reposition wurde bei allen Patienten erreicht. Postoperativ wurde eine sofortige Mobilisation bei 16 der 17 Patienten durchgeführt. Bei 1 Patienten zeigte sich postoperativ in der Röntgenkontrolle eine nichtdislozierte tiefe Kollumfraktur der
Gegenseite, nachdem operativ eine Trümmerfraktur des aufsteigenden Asts und der Gelenkfortsatzbasis der rechten Seite versorgt wurden. 2 Patienten mit Unsicherheiten bei der Okklusion wurden postoperativ für 5 Tage mit Führungsgummis behandelt. Weiche Kost wurde für 7 Tage postoperativ gegeben.

6 Monate nach dem Eingriff war bei allen Patienten die Mundöffnung mit $>40 \mathrm{~mm}$ SKD ohne Deviation und ohne Limitation, $>1 \mathrm{~mm}$ bei Laterotrusion und Protrusion nicht eingeschränkt. Schmerzen im Bereich der Kiefergelenke wurden nicht berichtet, und es lag keine Funktionseinschränkung des N. facialis vor. Die Länge der submandibularen Inzision wurde mit 4-5 cm gemessen. Die submandibulare Narbe war bei 2 Patienten nicht sichtbar und bei $7 \mathrm{~Pa}$ tienten ästhetisch akzeptabel.

\section{Diskussion}

Bei der konservativen Behandlung von dislozierten Kollumfrakturen können Einschränkungen der Kiefergelenkfunktion und eine Verkürzung des aufsteigenden Unterkieferasts mit offenem Biss und Okklusionsstörung zu unbefriedigenden Ergebnissen führen [2, 5]. Aufgrund der forcierten Adaption bei veränderter Kiefergelenkmorphologie zeigte sich in klinischen Langzeituntersuchungen nach konservativer Therapie eine Limitation bei der Mundöffnung mit Deviation und kompensatorisch habitueller Kiefergelenkluxation der kon-

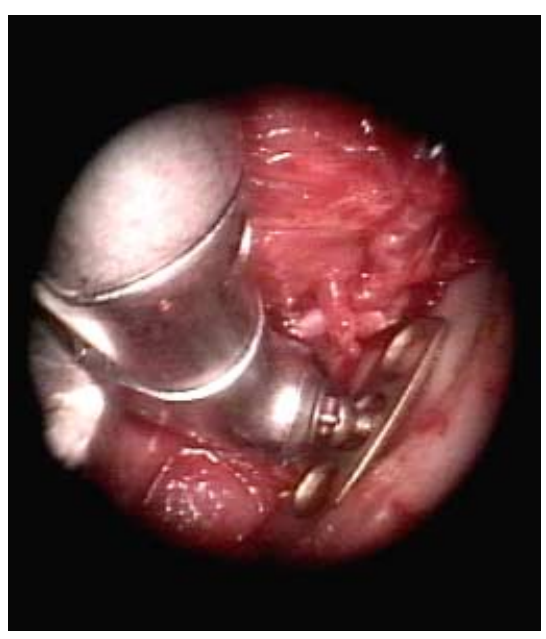

Abb. 4 Intraoperatives Bild von Abb. 3, abgewinkelter Schraubendreher zur rein transoralen Platteninsertion tralateralen Seite. Zur Vermeidung dieser Komplikationen wird die operative Frakturversorgung mit anatomisch regelrechter Reposition angestrebt $[3,4,5$, $7,9,22,23]$. Die Indikationen für die offene oder geschlossene Behandlung von dislozierten Kollumfrakturen werden in der Mund-, Kiefer- und Gesichtschirurgie kontrovers diskutiert, da bei der offenen Frakturreposition als Komplikation eine Schädigung des $\mathrm{N}$. facialis und Narben im sichtbaren Bereich beschrieben sind $[4,7,20,22,23]$.

Endoskopisch assistierte Techniken für unterschiedliche Indikationen im kraniomaxillofazialen Bereich wurden berichtet $[4,6,13,19]$. Endoskopisch assistierte Techniken mit limitierten extraoralen oder transoralen Inzisionen zur Minimierung des Risikos dieser Komplikationen wurden beschrieben $[1,8,11$, 12, 15]. Bei den 17 Patienten nach endoskopisch assistierter offener Reposition über eine submandibulare oder transorale Schnittführung waren die anatomische Reposition achsengerecht und die postoperative Kiefergelenkfunktion nicht eingeschränkt. Alle 17 Patienten zeigten eine regelrechte Okklusion ohne Einschränkung der Mundöffnung. Die Funktion des N. facialis war 6 Monate postoperativ nicht beeinträchtigt, und Narben im sichtbaren Bereich waren ästhetisch akzeptabel. 2 unterschiedliche operative Zugänge für die Behandlung von Kollumfrakturen wurden vorgestellt. Der operative Zugang wurde in Abhängigkeit vom Typ und von der Lokalisation der Kollumfrakturen gewählt. Der extraorale Zugang wurde bei Frakturen mit ausgeprägter Dislokation insbesondere nach medial und bei Trümmerfrakturen verwendet. Eine ausgezeichnete Übersicht im Bereich des Operationsfelds und ein gutes Handling der Instrumente für die Frakturreposition wurden beim submandibularen Zugang beobachtet $[11,12,15]$. Beim extraoralen Zugang wurde als positiv bewertet, dass bei komplizierten Frakturen mit erschwerter Reposition die Inzision erweitert und damit eine verbesserte Übersicht und erleichterte Frakturreposition erreicht werden können. Der transorale Zugang wird klinisch bei Kollumfrakturen nicht routinemäßig verwendet, da die Frakturreposition schwierig und die Übersicht transoral anatomisch bedingt durch den Proc. muscularis limitiert sein können. Durch 


\section{Originalien}

die Verwendung von gewinkelten Optiken werden eine verbesserte Übersicht erreicht und die transorale Reposition von Kollumfrakturen erleichtert [1, 8, 15]. Der transorale Zugang wurde zur endoskopisch assistierten Reposition von Kollumfrakturen mit Fragmentdislokation nach lateral durchgeführt. Bei stark dislozierten Frakturen mit Dislokation nach medial und bei Trümmerfrakturen ist der extraorale Zugang für die endoskopisch assistierte, operative Versorgung des Kollums indiziert.

Verglichen mit dem submandibularen Zugang ist der transorale Zugang weniger zeitintensiv. Intraorale Narben sind nicht im sichtbaren Bereich, und bei streng subperiostaler Präparation ist das Risiko für eine Schädigung des N. facialis minimal. Nichtdislozierte Kollumfrakturen und hohe intrakapsuläre Kollumfrakturen werden durch Immobilisation mittels IMF und frühfunktionelle Behandlung therapiert.

Vor der Durchführung endoskopisch assistierter Operationen ist ein intensives Training der endoskopischen Technik und der Handhabung des Instrumentariums Voraussetzung. Eine fachkundige Anleitung des Operationspersonals ist empfehlenswert. Aufgrund einer steilen Lernkurve kann der initial erhöhte Zeitaufwand deutlich reduziert werden. Abgewinkelte Bohrer und Schraubendreher haben sich bei der transoralen Frakturversorgung zur Vermeidung transbukkaler Stichinzisionen bewährt.

\section{Literatur}

1. Chen C-T, Lai J-P, Tung T-C, Chen Y-R (1998) Endoscopically assisted mandibular subcondylar fracture repair. Plast Reconstr Surg 103:60-65

2. Dahlstrom L, Kahnberg KE, LindahI L (1989) 5 yeas follow-up on condylar fractures. Int J Oral Maxillofac Surg 18: 18-23

3. Eckelt U, Gerber S (1981) Zugschraubenosteosynthese bei Unterkiefergelenkfortsatzfrakturen mit einem neuartigen 0steosynthesebesteck. Zahn Mund Kieferheilkd 69: 485490

4. Ellis E III, Dean J (1993) Rigid fixation of mandibular condyle fractures. Oral Surg Oral Med Oral Pathol 76:6-15

5. Ellis III E, Simon P, Throckmorton GS (2000) Occlusal results after open or closed treatment of fractures of the mandibular condylar process. Int J Oral Maxillofacial Surg 58:260-268

6. Graham HD, Spring P (1996) Endoscopic repair of frontal sinus fracture: case report.J Craniomaxillofac Trauma 2:4;52-55

7. Hall MB (1994) Condylar fractures: surgical management.J Oral Maxillofac Surg 52:11891192

8. Jacobovicz J, Lee C, Trabulsky PP (1998) Endoscopic repair of mandibular subcondylar fracture. Plast Reconstr Surg 101:437-441

9. Koberg W, Momma WG (1978) Treatment of fractures of the articular process by functional stable osteosynthesis using miniaturized dynamic compression plates. Int J Oral Surg 7: 256-262

10. Krenkel C (1992) Axial "anchor" screw (lag screw with biconcave washer) or "slantedscrew" plate for osteosynthesis of fractures of the mandibular condyloid process.J Craniomaxillofac Surg 20:348-353

11. Lauer G, Schmelzeisen R (1999) Endoscope-assisted fixation of mandibular condylar process fractures.J Oral Maxillofac Surg 57:36-39

12. Lauer G, Schmelzeisen R, Wichmann U (1998) 2. Endoskopgestützte Fixation von Gelenkfortsatzfrakturen des Unterkiefers. Dtsch Z Mund Kiefer Gesichtschir [Suppl 1] 2:68-70
13. Lee CH, Lee C, Trabulsy PP (1996) Endoscopicassisted repair of a malar fracture. Ann Plast Surg 37: 178-183

14. Schön R, Gellrich N-C, Schramm A, Schmelzeisen R (2000) Endoskopische Chirurgie im Mund-, Kiefer- und Gesichtsbereich - Videodemonstration einer endoskopisch assistierten Versorgung einer dislozierten Jochbeinfraktur. J DGPW 12:21;12

15. Schön R, Gutwald R, Schramm A, Gellrich N-C, Schmelzeisen R (2000) Endoscopic assisted treatment of condylar fractures. Differential indication of the extraoral and intraoral approach. The Congress 2000 of the EACMFS J Craniomaxillofac Surg [Suppl 3] 28:85

16. Schön R, Roveda SIL, Carter B (2001) Mandibular fractures in Townsville, Australia. Incidence, etiology and treatment using the $2.0 \mathrm{AO} / \mathrm{ASIF}$ miniplate system. Br J Oral Maxillofac Surg 39: 145-148

17. Schuchardt K, Metz HJ (1976) Injuries of the facial skeleton. Mod Trends Plast Surg 2:62-107

18. Tasanen A, Lamberg MA (1976) Transosseous wiring in the treatment of condylar fractures of the mandible.J Maxillofac Surg 4:200-206

19. Vasconez LO, Core GB, Oslin B (1995) Endoscopy in plastic surgery:an overview. Clin Plast Surg 22:585-589

20. Walker RV (1994) Condylar fractures: nonsurgical management.J Oral Maxillofac Surg 52: 1185-1188

21. Weinberg MJ, Merx P,Antonymshyn 0, Farb R (1995) Facial nerve palsy after mandibular fractures. Ann Plast Surg 34:546-549

22. Widmark G, Bagenholm T, Kahnberg KE, Lindahl L (1996) Open reduction of subcondylar fractures. Int J Oral Maxillofac Surg 25: 107111

23. Zide MF, Kent JN (1983) Indications for open reduction of mandibular condyle fractures.J Oral Maxillofac Surg 41:89-98 